\title{
La Microscopía Electrónica de Barrido para el estudio del Patrimonio Histórico. Nueva sección del Departamento de Análisis
}

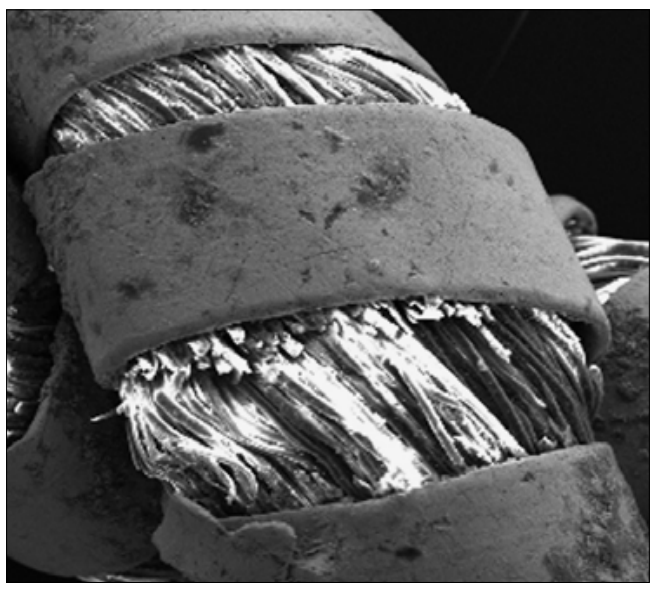

Rosario Villegas Sánchez

Asesor Técnico del Departamento de Análisis

Centro de Intervención del IAPH

\section{Resumen}

El Instituto Andaluz del Patrimonio Histórico ha creado la Sección de Microscopía Electrónica de Barrido (siglas en inglés SEM) dentro del Departamento de Análisis, lo cual permite ampliar y agilizar la realización de análisis para identificación de materiales y el estudio morfológico de muy diversas muestras. El equipo instalado es el modelo JEOL Jsm-5600LV con microanalizador Oxford modelo INCA Energy 200, y el equipo de preparación de muestras Bal-Tec SCD-005.

Para alojar dicha Sección se han llevado a cabo obras de ampliación del Departamento de Análisis, con las que se han dotado todas las instalaciones técnicas necesarias para el funcionamiento del equipo.

\section{Palabras clave}

SEM / MEB / LV-SEM / Microanálisis / EDX / Patrimonio Histórico

\section{INTRODUCCIÓN}

La microscopía, en general, es un conjunto de técnicas que permiten ver objetos muy pequeños, no observables a simple vista. La resolución de un microscopio, es decir, el tamaño del objeto más pequeño que es posible distinguir, es proporcional a la longitud de onda de la radiación utilizada para iluminar la muestra. La primera en desarrollarse fue la microscopía óptica, que utiliza la luz visible; dada la longitud de onda de esta radiación, sólo pueden apreciarse partículas de tamaño superior a unas decenas de micras $\left(10^{-5} \mathrm{~m}\right)$.

La microscopía electrónica empezó a utilizarse, en el modo de transmisión, en los años 30 y posteriormente en los 60 se construyeron los primeros microscopios de barrido comerciales. Actualmente la resolución del SEM es del orden de $40 \AA$, pudiéndose obtener aumentos teóricos de 300.000x. Esta es una de sus ventajas sobre la microscopía óptica, siendo otras la gran profundidad de campo, es decir, mantener enfocados puntos con alturas muy diferentes, y la visión estereoscópica de las muestras.

\section{OBRAS DE INSTALACIÓN}

El alojamiento de la sección de microscopía electrónica se ha efectuado en una zona anteriormente sin uso dentro del edificio principal del Instituto. Se trata de un área residual de una de las dos salas de expo- 
Figura I. Planta de la Sala de Microscopía electrónica

Figura 2. Corte vertical de las instalaciones siciones, concretamente la que se encuentra debajo de las oficinas de Dirección y Administración. Esta sala se extiende hacia el Este y el Sur, ocupando parte de la crujía occidental del Claustro de Legos de la Cartuja de Santa María de las Cuevas, hasta llegar a las actuales dependencias del Departamento de Análisis. La zona donde se ha ubicado tiene una superficie de $20 \mathrm{~m}^{2}$ y una altura libre de 7,10 m, con diversos huecos y ventanas tanto en planta baja como en la segunda.

El programa desarrollado se compone esencialmente de los siguientes ámbitos:

- Sala de microscopía electrónica y microanálisis, capaz para contener dichos equipos y para la estancia habitual de una/dos personas.

- Zona de despacho para uso de dos personas, que se ha construido en planta alta aprovechando la doble altura de dicho espacio.

- Espacios para almacenamiento del recipiente de nitrógeno líquido y botella de nitrógeno a presión y para el alojamiento de la maquinaria de acondicionamiento de aire, así como la disposición de los correspondientes cuadros eléctricos.

El diseño general del conjunto, debido a las características de su ubicación, ha perseguido que su repercusión visual exterior sea prácticamente nula.

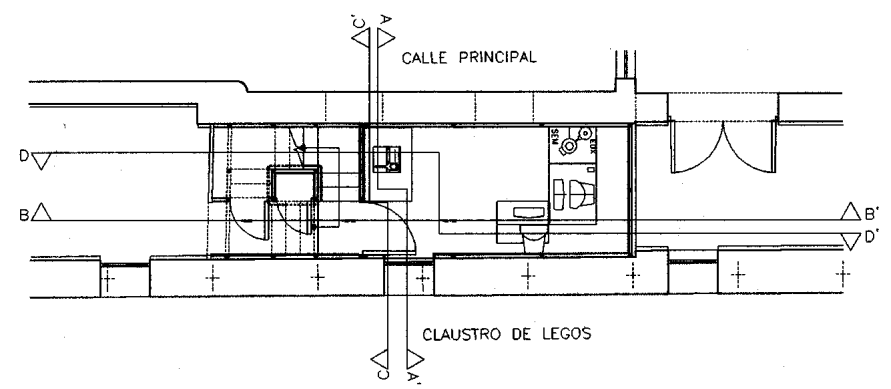

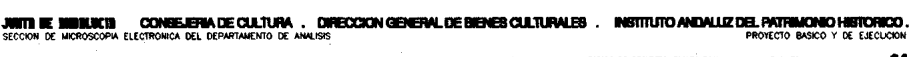

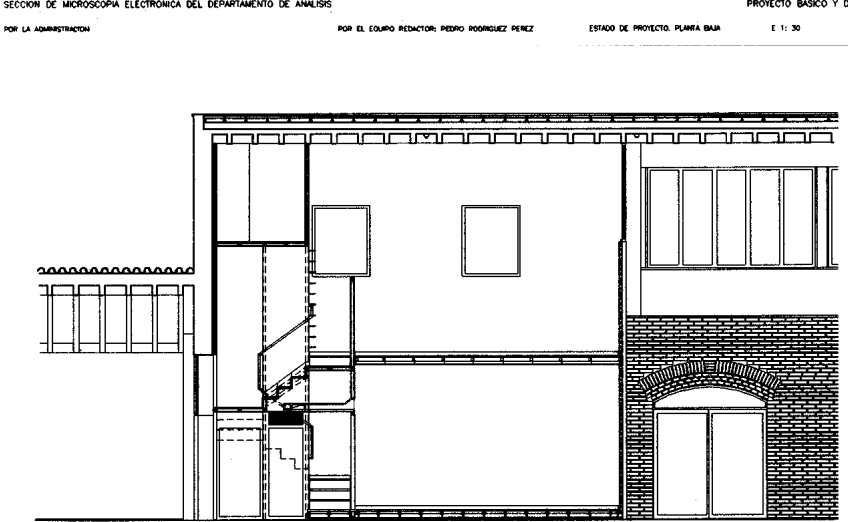

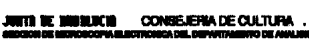

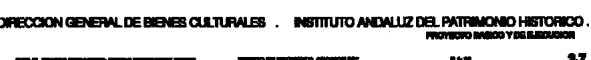

Constructivamente se ha procurado minimizar el impacto de la intervención, para lo cual se ha realizado una estructura metálica ligera autosustentable, a base de perfiles soldados o atornillados, que va apoyada sobre la solería original previa disposición de unos durmientes de tablero de partículas de madera aglomerada con cemento. De este modo, los anclajes a las masas murales y demás fábricas preexistentes son exclusivamente puntuales y de carácter secundario.

Estos tableros de partículas con aglomerante cementicio presentan una excelente estabilidad dimensional, resistencia mecánica y contra el fuego, habiéndose empleado tanto como elemento estructural, constituyendo el suelo de diversas zonas, como de revestimiento de distintas paredes.

La sala de microscopía se ha dotado de un falso suelo técnico, para facilitar la ejecución y el mantenimiento de la red de refrigeración del microscopio electrónico, así como las de alimentación eléctrica de los distintos equipos. Se han instalado paneles absorbentes acústicos en las paredes, preparados perforando los tableros lisos de empleo general y trasdosándolos con lana de roca.

La instalación de aire acondicionado está compuesta de un acondicionador-bomba de calor condensado por aire y una red de conductos de impulsión fabricados con paneles de fibra de vidrio forrado exteriormente con aluminio, siendo el retorno por plenum. La alimentación de energía eléctrica se efectúa a partir de un cuadro principal, del que se alimenta a su vez otro secundario (máquinas térmicas). El primero de los cuadros tiene toma de tierra independiente del resto del edificio. La iluminación artificial se resuelve con luminarias dotadas de lámparas fluorescentes con reactancias electrónicas.

Se han extendido hasta estos locales las redes ya existentes de voz y datos, detección de intrusismo y alarma de incendios.

Nota: Tomado del Proyecto Básico y de Ejecución "Adaptación parcial de la crujía oeste del Claustro de Legos para su uso como Sección de microscopía electrónica del Departamento de Análisis", redactado por Pedro Rodríguez Pérez y $M^{a}$ Luisa Marín Martín, arquitectos, y Carmen Ferrera Sánchez, arquitecto técnico. La obra ha sido realizada por la empresa Joaquín Pérez Construcciones.

\section{FUNDAMENTOS DE LA MICROSCOPÍA ELECTRÓNICA DE BARRIDO}

La microscopía electrónica se basa en dos hechos. Por una parte, De Broglie, en 1924, postuló la dualidad onda-partícula: a una partícula en movimiento se le puede asociar una onda, cuya longitud depende de su masa y de su energía. En el caso de los electrones, esta longitud es mucho menor que la de la luz visible, 
por lo que el poder de resolución de un microscopio que utilice electrones como radiación de iluminación es mayor que el de uno óptico.

Posteriormente, en 1926 Brusch demostró que se pueden emplear campos eléctricos y magnéticos como lentes para controlar un haz de electrones $u$ otras partículas con carga, comenzando así la óptica electrónica.

En 1939 se construyó el primer microscopio electrónico de transmisión con una resolución 100 veces superior a uno óptico. Después de la II Guerra Mundial el desarrollo ha sido muy rápido, primero con los de transmisión de alta resolución y a partir de 1965 los de barrido.

\section{Configuración del microscopio electrónico de barrido}

El elemento más importante del equipo es la columna en la que se produce y se mueve el haz de electrones. Los electrones se obtienen, en el caso más frecuente, por calentamiento de un filamento y se extraen de él por la atracción de un campo eléctrico débil. El filamento puede ser de wolframio o hexafluoruro de lantano. Los electrones que se obtienen así se aceleran posteriormente para su paso por la columna bajo la acción de una diferencia de potencial que puede tener un valor máximo de 30.000 voltios. En toda la columna debe hacerse un alto vacío, para evitar que los electrones choquen con los átomos de gas que pudiesen existir en dicho espacio.

Los electrones salen del cañón como un haz divergente que se focaliza y condensa por medio de una serie de lentes magnéticas y aperturas. Al final de la columna existe un dispositivo magnético que induce en el haz el movimiento de barrido sobre la muestra. Las lentes de objetivo enfocan el haz para conseguir que tenga la sección más pequeña posible al incidir sobre la superficie de la muestra. Ésta está colocada sobre un soporte que puede moverse de diferentes formas (rotación, inclinación y traslación en los tres ejes) para observar cualquier punto de la muestra y con distinta inclinación.

La cámara donde se coloca la muestra, igual que el cañón, se encuentra en vacío. En los SEM convencionales, este vacío debe alcanzar valores muy altos, del orden de $10^{-4} \mathrm{~Pa}$; en los modelos de última generación, denominados de bajo vacío o presión variable, la presión de trabajo en la cámara de muestra en este modo puede ser hasta de $270 \mathrm{~Pa}$.

Cuando los electrones inciden sobre la muestra se producen diversas interacciones, que dan lugar a una serie de fenómenos, que pueden utilizarse con distintos fines analíticos. Para ello, en la cámara se instalan diversos detectores que recogen los distintos tipos de señal que se producen. En la figura 3 se representan las distintas señales emitidas por la muestra, siendo las más utilizadas las siguientes.
I.- Los átomos que forman la muestra emiten electrones secundarios como consecuencia del choque del haz de electrones. Estos electrones secundarios tienen poca energía, por lo que sólo pueden proceder de una fina capa superficial y son los que se usan para la observación de la morfología y topografía de la muestra (microscopía propiamente dicha). Esto se debe a que la cantidad de electrones secundarios emitidos por la muestra depende mucho del ángulo de incidencia del haz de electrones y, por tanto, de su relieve superficial.

2.- Los electrones del haz penetran en la muestra y después de ceder parte de su energía a ésta retroceden y salen de la superficie; se produce la emisión de electrones retrodispersados, de muy alta energía, que proceden de zonas más internas. La producción de electrones retrodispersados está relacionada con el número atómico de los átomos que forman la muestra, de manera que este haz se puede utilizar para obtener datos sobre la composición elemental de la muestra. Normalmente se emplea produciendo imágenes con distintos tonos de gris que informan sobre la composición de cada zona.

3.- Las transiciones de electrones que se producen en los átomos de la muestra provocan la emisión de Rayos X, cuya energía es característica de los elementos presentes en ella, por lo que se pueden utilizar para identificar dichos elementos; pero además, la cantidad de fotones de cada energía emitidos es proporcional al número de átomos de cada tipo existentes. Por lo tanto, es posible realizar un análisis cualitativo (para elementos de número atómico superior a 10) y semicuantitativo (con una precisión aproximada del 10\%). Como el haz de electrones se puede enfocar de forma muy fina, es posible analizar áreas muy pequeñas, incluso puntuales.

La formación de imágenes no es comparable a la de una imagen óptica, sino que se construye a partir de las señales que emergen de la muestra. En los diferentes detectores se convierten en señales eléctricas

Figura 3: Interacciones del haz de electrones con la muestra

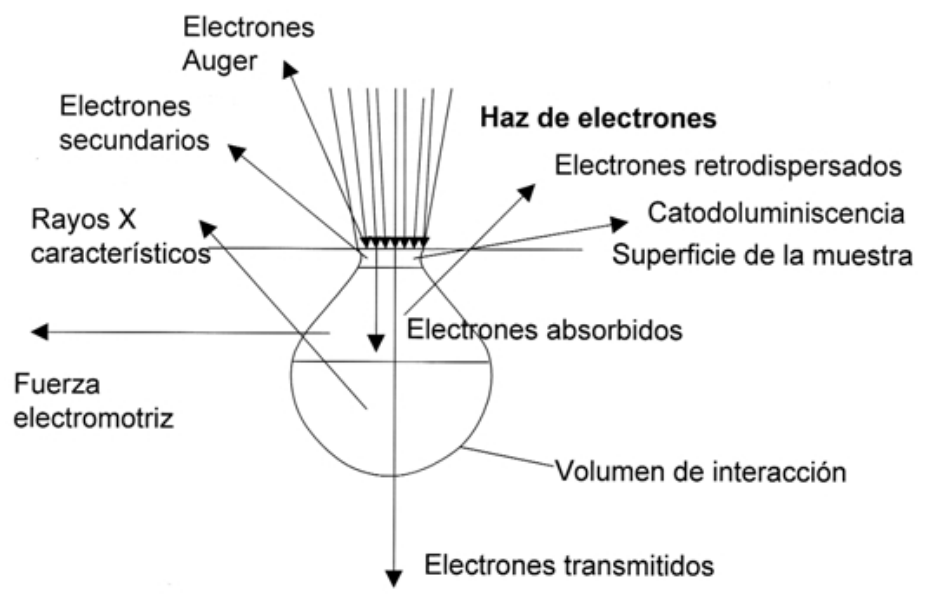


Figura 4. Microscopio electrónico del IAPH (Fotografía Eugenio Fernández)

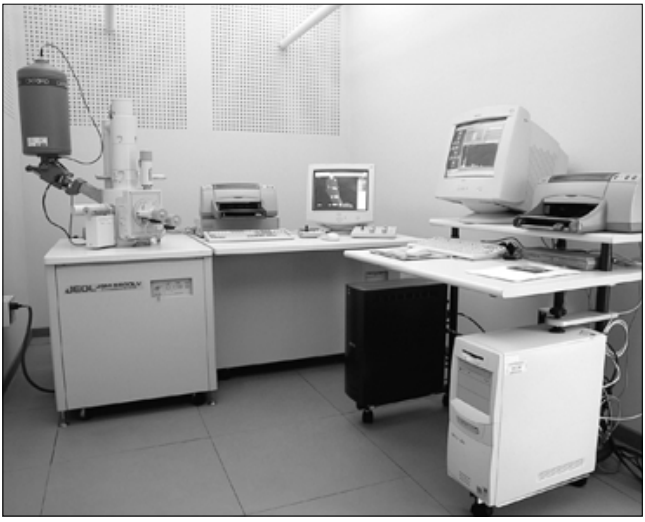

que, a través de un amplificador, se llevan a un tubo de rayos catódicos donde se produce la imagen (de forma parecida a la televisión convencional). Así pueden obtenerse distintas imágenes según la señal que se esté utilizando, pudiéndose incluso combinar dos tipos de señal. Actualmente la imagen también se digitaliza para su posterior tratamiento. El aumento con que se observa la imagen se obtiene en función de la relación entre el tamaño de la zona que barre el haz de electrones en la muestra y el de la imagen en el tubo de rayos catódicos; cuanto menor sea la zona barrida mayor es la amplificación.

\section{Preparación de muestras}

Dada la gran variedad de aplicaciones de la microscopía electrónica de barrido, existen numerosos tipos de muestras que pueden estudiarse, cada uno de los cuales requiere distintas técnicas de preparación. En todos los casos se deben tener en cuenta tres consideraciones.

La superficie debe estar limpia, ya que esto es lo que se observa. Debe conservarse la morfología original; si se estudia una muestra que contenga agua o gas, al someterla a vacío puede encogerse o deformarse, por lo que las muestras biológicas deben fijarse, deshidratarse o secarse. Por último debe evitarse que las muestras se carguen electrostáticamente; el haz de electrones que irradia la muestra en su mayor parte se acumula en su superficie y si ésta no es conductora se carga. Para evitar esto, se puede recurrir a tres modos de trabajo con muestras no conductoras: se recubren con algún material que sí lo sea (oro o carbono), se observan con un voltaje de aceleración bajo o se utiliza el modo de bajo vacío. Cuando se recubren las muestras, debe procurarse que el espesor del recubrimiento no sea excesivo, ya que se perderían detalles de la superficie.

En el Departamento de Análisis se ha instalado un equipo de metalización de muestras, marca Bal-Tec modelo SCD-005 con la unidad de sombreado de carbón modelo CEA-035. Con él se procede a la preparación de muestras no conductoras (madera, estratigrafías de películas pictóricas, piedra, morteros, etc.), que son una gran parte de las analizadas. Consta de dos partes, una para recubrir con oro y otra para carbono.

\section{MICROSCOPÍA DE BAJO VACÍO (LV SEM)}

Este modo de trabajo se está instalando en los microscopios de barrido más recientes. En el sistema convencional, tanto el cañón como la cámara de muestra se mantienen a la misma presión, alto vacío. En los equipos que incorporan el método de bajo vacío o presión controlada se instala un sistema de bombas de vacío adicional, de manera que una segunda forma de trabajo consiste en mantener una presión más alta (entre I y $270 \mathrm{~Pa}$ ) en la cámara de muestra que en la columna. En estas condiciones las moléculas del gas que rodea la muestra se ionizan por los choques con los electrones y consiguen disipar la carga que se acumula en ésta; de esta forma, es posible observar un material no conductor sin necesidad de metalizarlo. Así mismo, se pueden estudiar muestras con agua o con otro tipo de fluido.

Con este modo de trabajo sólo es posible detectar los electrones retrodispersados y no los secundarios, ya que debido a su baja energía son absorbidos y frenados por las moléculas del gas. Con el detector de retrodispersados es posible obtener tres tipos de imágenes, la composición, es decir, diferentes tonos de gris según la composición elemental de la muestra, la topográfica, el relieve de la superficie, y la combinación de ambas.

El equipo que se ha instalado en el IAPH es un modelo JEOL JSM-5600LV. Es un equipo de presión variable, siendo las condiciones de trabajo en cada modo las siguientes:

Alto vacío: Presión $7 \times 10^{-4} \mathrm{~Pa}$, utilizando una bomba difusora y una rotatoria de dos etapas.

Bajo vacío. Presión 6 a 270 Pa, ajustable manual o automáticamente, empleando otra bomba rotatoria independiente.

El voltaje de aceleración es de 0,5 a 30 kV, pudiéndose ajustar desde 500 a $3.000 \mathrm{~V}$ en pasos de $100 \mathrm{~V}$ y desde 3 a $30 \mathrm{kV}$ en pasos de I kV. La platina portamuestras permite la instalación de diversos portamuestras y está dotada de movimiento en los tres ejes, de rotación y de inclinación. Tiene cuatro velocidades de barrido.

La resolución de imagen es de 3,5 nm para electrones secundarios y $5,5 \mathrm{~nm}$ para electrones retrodispersados, siendo los aumentos de 18 hasta 300.000 . Los detectores instalados son de electrones secundarios y de electrones retrodispersados, con el que se obtienen tres tipos de imagen, de composición, topográfica y la mezcla de ambas.

El control se realiza con ordenador y sistema operativo Windows NT, estando también equipado con un teclado auxiliar convencional. Se pueden grabar las condiciones de trabajo, hasta 20 usuarios con 100 procedimientos cada uno.

\section{MICROANÁLISIS POR ENERGÍAS DISPERSIVAS DE RAYOS $X$}

Uno de los detectores que pueden instalarse en la cámara de muestras es el espectrómetro de energías dis- 
persivas de Rayos $X$, que también puede emplearse en el modo de bajo vacío. La energía de los Rayos $X$ que emiten los distintos átomos presentes en la muestra es característica de cada elemento; su detección y el posterior tratamiento de estas señales hacen posible la identificación de la composición elemental de la muestra.

La espectrometría de energías dispersivas es actualmente la técnica de microanálisis más utilizada. Como detector se usa un semiconductor de Si(Li) que capta los Rayos $X$ emitidos por la muestra, transmitiendo una señal eléctrica proporcional a la energía de la radiación a un amplificador, del que pasa a un analizador multicanal. Este analizador debe calibrarse previamente con una muestra patrón, de manera que posteriormente puedan identificarse los elementos presentes en la muestra. El detector $\mathrm{Si}(\mathrm{Li})$ debe mantenerse a una temperatura muy baja, para lo cual se emplea nitrógeno líquido.

El tratamiento informático de las señales permite la realización de análisis de diferentes zonas de la muestra: toda la superficie que se esté observando, una línea, un área seleccionada, o un punto. También es posible obtener los mapas de distribución de un elemento determinado en la superficie de la muestra, y combinar mapas correspondientes a distintos elementos para conseguir información sobre la distribución de compuestos.

El sistema de microanálisis por energías dispersivas de Rayos $X$ que se ha instalado en el IAPH es el modelo Oxford INCA Energy 200. Está constituido por un detector de Si(Li) con un área de detección de $10 \mathrm{~mm}^{2}$ y una resolución de 138 eV, pudiéndose detectar desde Berilio a Uranio. Se puede realizar análisis de área, de línea o puntual. Los espectros adquiridos pueden compararse entre sí o con referencias y los picos se identifican y etiquetan automática o manualmente. Igualmente pue-
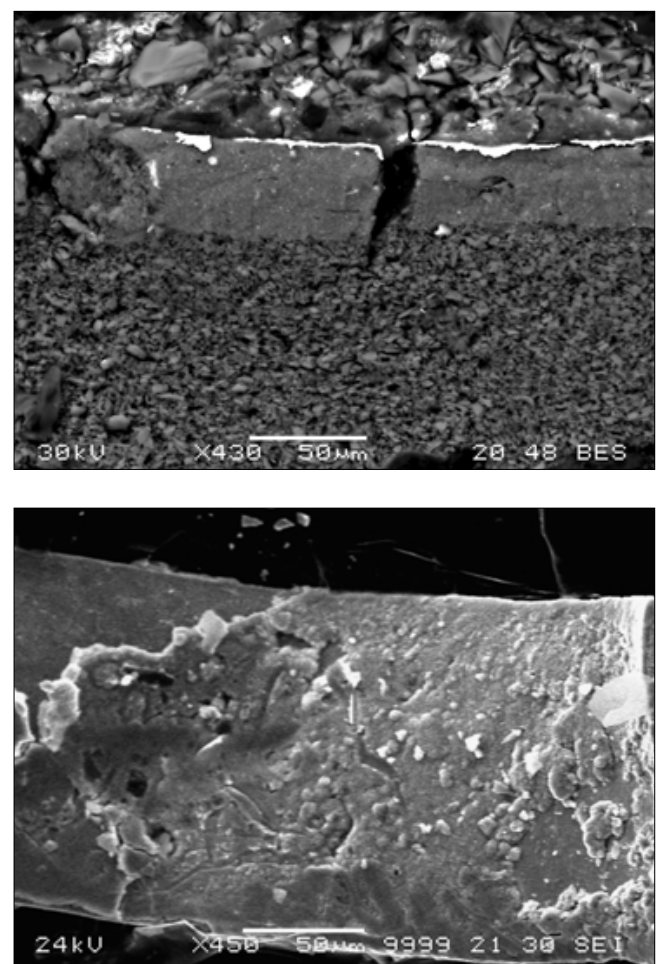

den realizarse mapas de distribución de elementos, combinando los mapas individuales. Se controla a través de Windows NT, con el software INCA Energy 200.

\section{APLICACIONES DE LA MICROSCOPÍA ELECTRÓNICA DE BARRIDO AL ESTUDIO DE LOS BIENES CULTURALES}

La microscopía electrónica de barrido se emplea para el estudio morfológico y/o el análisis elemental de numerosos materiales constitutivos de bienes culturales, de productos y agentes de deterioro o de tratamientos de conservación.

El microanálisis permite identificar pigmentos y cargas en películas pictóricas a partir de sus composición elemental, pudiéndose observar también las características texturales de las distintas capas que forman la policromía: tamaño de los granos del pigmento, espesor de las capas, mezclas de pigmentos, etc.

En los objetos textiles es muy útil para el estudio de diversos materiales, como hilos y otros elementos metálicos, en los que se puede estudiar la forma de elaboración del hilo, la composición del metal o las alteraciones que presente.

En el caso de los materiales pétreos y similares (cerámica, piedra, mortero, etc.), así como sus productos de alteración (sales solubles, eflorescencias, costras de reacción con la atmósfera, etc.), puede obtenerse la composición química de los minerales que los forman, incluso de aquellos que estén en proporción muy baja y no puedan detectarse por técnicas como la difracción de Rayos $X$, ya que el mi-

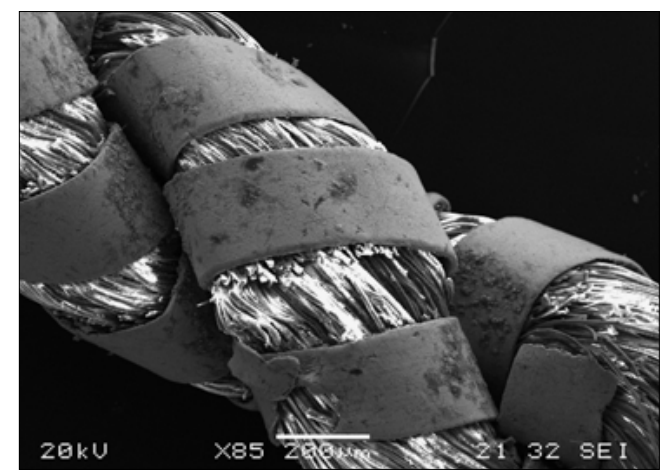

Figura 5. Estratigrafía de pintura Figura 6. Hilo metálico

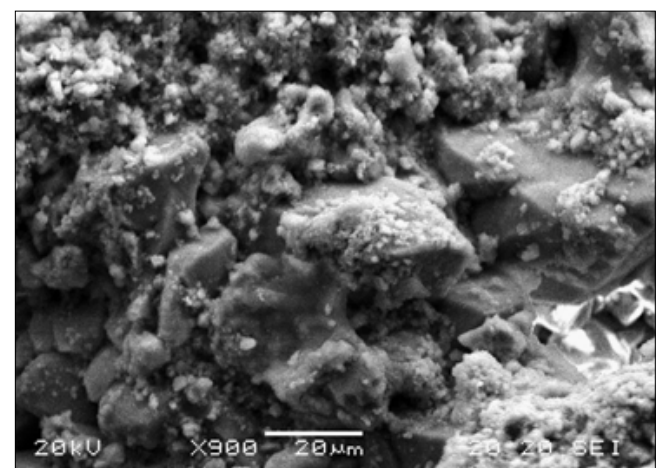



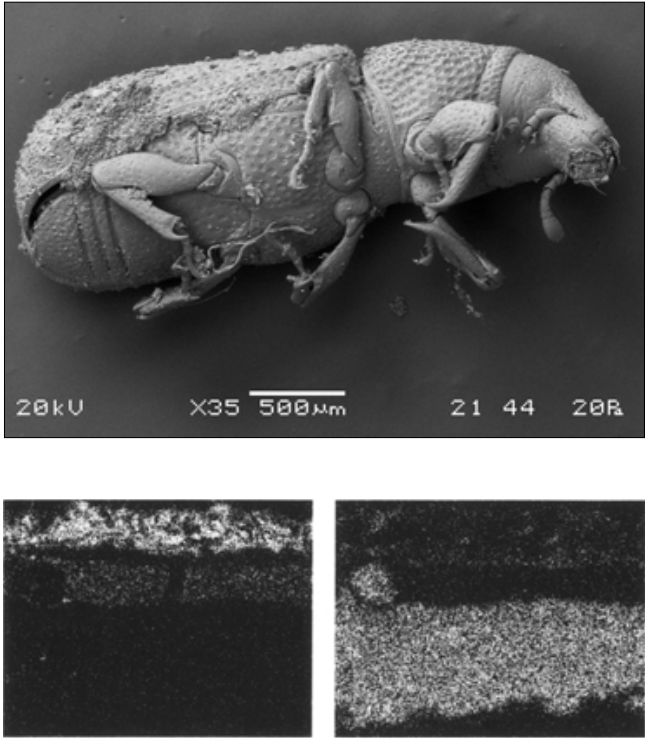

Slicon Ka1

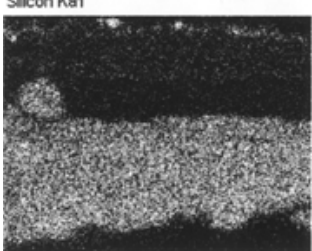

Figura 9. Insecto

Figura 10. Mapa de distribución de elementos en una estratigrafía

Figura II. Microanálisis de policromía

\section{Calcium Ka1}

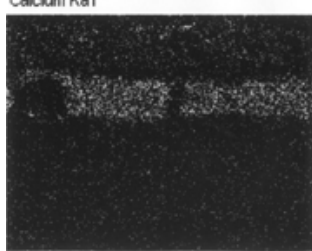

Aluminum Ka1

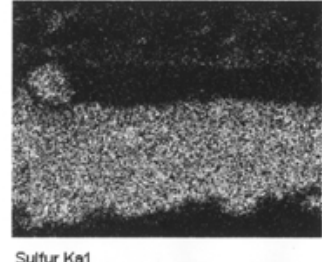

Sulfur Ka1

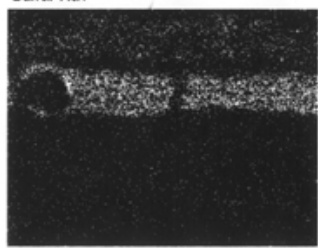

Iron Ka1

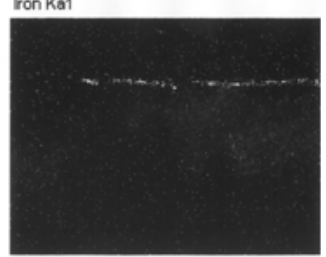

Gold La1

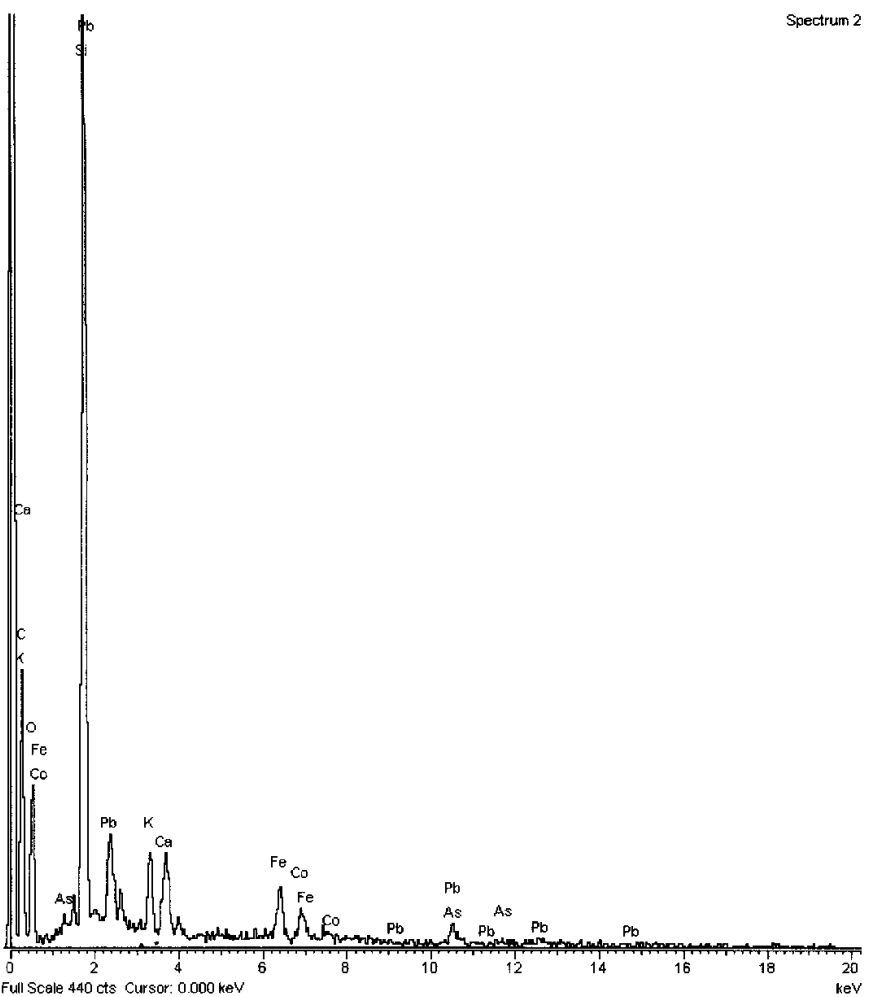

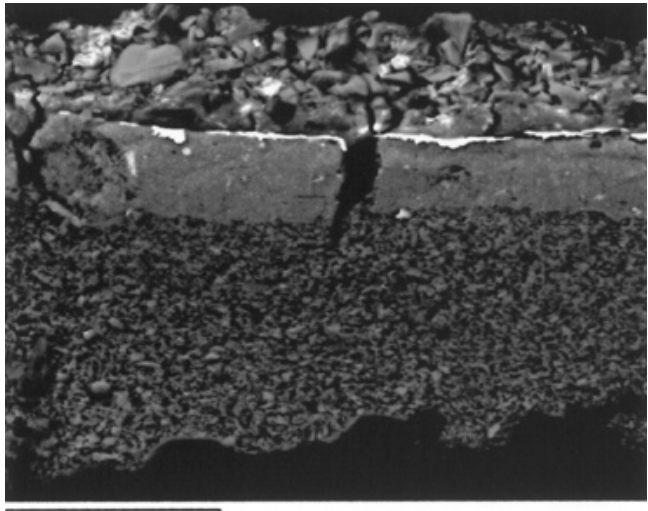

$100 m$

Bloctron imago 1

croanálisis puede realizarse en un punto. Combinando la observación de las formas cristalinas que se presenten con el microanálisis pueden identificarse numerosos componentes minerales.

Similar es la aplicación en el caso de los metales, pudiéndose obtener la composición de las aleaciones y observar la estructura metalográfica: tamaño de grano, porosidad, etc., así como de sus productos de corrosión. La preparación de las muestras en forma de sección perpendicular a la superficie permite observar las diferentes capas de corrosión que existan, su textura y composición.

Otras aplicaciones posibles serían la caracterización de tintas, identificación de maderas o carbones, la identificación de pólenes, el estudio de huesos y otros restos biológicos en yacimientos arqueológicos o paleontológicos.

Por lo que se refiere al estudio de tratamientos, es posible estudiar el efecto de distintos tipos de tratamientos en diversos materiales: la eficacia de tratamientos biocidas observando los microorganismos después de la aplicación, o bien sus efectos secundarios sobre el sustrato a través de la aparición de posibles subproductos. Los tratamientos consolidantes o hidrófugos para piedra pueden estudiarse perfectamente ya que forman una película superficial cuyas características (recubrimiento de los granos minerales, penetración en la piedra, uniformidad de su distribución) son indicativas de su posible comportamiento frente a los agentes de deterioro.

En definitiva, la microscopía electrónica de barrido es una herramienta muy poderosa cuyas aplicaciones al campo del Patrimonio Histórico son amplísimas, quedando aquí recogidos tan sólo algunos ejemplos.

\section{AGRADECIMIENTOS}

Las microfotografías y microanálisis de las figuras 5 a I I han sido realizados por Lourdes Martín García, Dra. Química del Departamento de Análisis del IAPH. 